\title{
O Papel da Certificação do Profissional de TI na Qualidade do Software: uma Investigação Preliminar
}

\author{
Alessandro Nunes de Souza, Edimara Mezzomo Luciano \\ Pontifícia Universidade Católica do Rio Grande do Sul \\ e-mail: ansouza@pucrs.br, eluciano@pucrs.br
}

\begin{abstract}
Resumo
Desenvolver software com qualidade é muitas vezes considerado ilusório, dada a dificuldade de mensuração neste sentido. Além desta abordagem tradicional de determinar se o software é bom ou não, têm surgido outras abordagens alternativas para a determinação da qualidade do software, destacando-se hoje a determinação da maturidade do processo e o reconhecimento, via certificação, dos profissionais de desenvolvimento de software. O objetivo deste artigo é mapear os principais testes de certificação profissional do mercado, listando características e explorando a relação custo-benefício, fornecendo subsídios para uma compreensão sistêmica da carreira do profissional de TI, principalmente na questão da qualidade do software por ele produzido. Através de uma pesquisa exploratória e descritiva, obteve-se como resultado um mapeamento das principais certificações do mercado: tipo, nível, características, testes, custos de treinamento e de exames, salário médio do certificado e a relação custo-benefício. Estes resultados podem contribuir: para as universidades, contribuindo para melhorias na formação dos egressos em áreas de TI; para os profissionais, para que possam compreender suas carreiras como um todo; para os gestores de programas de capacitação, para que reflitam sobre a estrutura dos programas de capacitação que promovem; e para a área de TI, através do aprofundamento do tema e identificação de novos focos de pesquisa, bem como a questão da influência da certificação profissional como pilar da qualidade de software.
\end{abstract}

Palavras-chave: certificação profissional; qualidade de software; profissional de TI

\begin{abstract}
To develop software with quality is sometimes considered illusive due to hardness of metrics in this topic. In addition to this traditional approach to determine software goodness, there are alternative approaches to determine the software quality, highlighting today the process maturity assessment and the recognition, via certification, of the software professionals. The goal of this article is to map the main tests of professional certification in the market, with characteristics and cost-benefit relation, supplying subsidies to a systemic understanding of IT professional career, related to the question of software with quality produced by him. Through an exploratory and descriptive research, a map of the main certifications in the market was gotten: type, level, characteristics, tests, costs of training and examinations, average wage of certified professional and costbenefit relation. These results can contribute: for universities, contributing for improvements in the formation of the egresses in IT areas; for professionals, to understand its careers as a whole; for qualification programs managers, to reflect the structure of the qualification programs they promote; and for information systems area, through the deepening of the subject and identification of new research needs, as well as the question of the influence of the professional certification as pillar of the software quality.
\end{abstract}

Key words: professional certification; software quality; IT professional

\section{Introdução: tema, justificativa e objetivo da pesquisa}

Desenvolver software com qualidade é por vezes considerado ilusório, dada a dificuldade de mensuração precisa neste sentido. Uma razão do porquê é difícil medir a qualidade do software vem das muitas deficiências teóricas e práticas do teste de software [29]. Este desafio tem levado muitas pessoas na comunidade de software a pensar que a determinação da qualidade de um produto de software é impraticável no dia-a-dia do mercado. Além da abordagem tradicional de determinar se o software é bom ou não, isto tem levado a abordagens alternativas para a determinação da qualidade do software. Segundo Basili e Zelkowitz [19], existem cinco fatores que influenciam a produtividade do software: fatores humanos, fatores do problema, fatores do processo, fatores do produto e fatores relacionados a recursos. Contudo, as duas abordagens-chave competindo atualmente são a determinação da 
maturidade do processo (por exemplo, através de CMM e CMMI) e o reconhecimento, via certificação, dos profissionais de software [29].

$\mathrm{Na}$ área de Tecnologia da Informação (TI), o curso de graduação já não é mais suficiente, em especial nos setores que fazem uso intensivo de softwares especializados [13]. Nestes setores, surge outro item na formação: a certificação, criada com o objetivo de atestar o que um profissional realmente sabe sobre uma determinada ferramenta ou tecnologia $[17$, 20]. Os fornecedores de TI oferecem certificações de seus produtos, publicações da área divulgam pesquisas que demonstram a necessidade de especialistas certificados, e os profissionais discutem os benefícios da certificação [6]: é uma cadeia que se forma, envolvendo uma série de aspectos a serem considerados por desenvolvedores, usuários, terceirizados e terceirizadores, profissionais de TI e de RH, gestores, enfim, por todos os envolvidos (direta ou indiretamente) com TI. O intuito por trás da certificação das habilidades do profissional é simples: não se deve deixar que um cliente final, não treinado para identificar pontos-chave do serviço prestado, determine se um candidato é qualificado para desempenhar os serviços desejados [29].

Um estudo de McKillip e Cox [14] feito com 21 supervisores de uma empresa de TI aponta que foi encontrada relação direta entre a certificação e a competência. Junto a isso, Lysaghta e Altschuldb [12] confirmam a importância de pesquisas sobre certificação, pois ela envolve a validação das competências, que são fundamentais para empresas de TI, cuja matéria-prima é o conhecimento. Com esta motivação em mente, o tema deste trabalho é a certificação profissional na área de TI, tendo como objetivo fazer um mapeamento dos principais testes de certificação profissional disponíveis no mercado, elencando características e explorando a relação custo-benefício dos mesmos. Desta forma, pretende-se fornecer subsídios para uma compreensão sistêmica da carreira do profissional de TI e para reflexões sobre a estrutura de programas de capacitação.

\section{Embasamento teórico}

Num mercado de trabalho cada vez mais competitivo, diferenciar-se é fundamental para que o profissional obtenha mais chances de se manter no mercado [3] fazendo o que gosta e sendo bem recompensado por isso [26]. Neste contexto, a certificação tem sido tema recorrente entre os profissionais de TI [6], sendo uma forma de estar à frente dos demais profissionais [14, 20]. A falta de competência e talento profissional na indústria de software é um dos maiores riscos de projeto, e o treinamento e a certificação trazem mudanças no conhecimento, habilidades, atitudes e comportamento social dos profissionais em um trabalho em particular [9].

O número de certificações tem aumentado muito na última década, em grande parte pela crescente onda de terceirização dos serviços de TI [13]. A certificação não é útil apenas para o profissional qualificado, mas também para o empregador, para o fabricante da tecnologia e para o usuário desta. Mulkeym e O'Neil JR [17] citam dois elementos que afirmam a importância da certificação: aspectos econômicos e ambiente de trabalho. Na visão econômica, a certificação ajuda as organizações a selecionar e manter mão-de-obra efetiva e produtiva, servindo às necessidades dos empregadores. No ambiente de trabalho, os autores citam que ele ficou menos rotineiro, exigindo, com isso, habilidades de pensamento de ordem mais elevada.

Para o empregador, a certificação ajuda a identificar mais facilmente o profissional com um certo conhecimento [8], podendo ainda diminuir o turnover, pois o profissional certificado em uma tecnologia tem um escopo de atuação mais focado - o que não deixa de ser um escopo menor [18], porque aumenta a motivação e deixa claro ao profissional como é a carreira que ele escolheu [13]. Para a empresa usuária da tecnologia certificada, cada 
programa de certificação dá uma melhor compreensão do que é atribuído ao funcionário de uma certa função [20].

O desenvolvedor da tecnologia tem interesse na certificação pois esta o diferencia das demais empresas (lhe confere um certo status), funciona como ferramenta de marketing (pois a certificação acaba gerando notícia), e porque a certificação dá uma garantia de que seus produtos estarão sendo utilizados em sua plenitude [18]. Para o profissional, a certificação amplia as suas chances de contratação e permanência no emprego, além de ser necessária para alguns tipos de concursos públicos.

O tema certificação é multifacetado. Para abordá-lo adequadamente é necessário, além dos aspectos técnicos, considerar também aspectos da carreira do profissional e do mercado.

\subsection{Certificação e competências em TI}

De acordo com Mckillip e Cox [14], as certificações profissionais estão crescendo em importância no mercado de trabalho, sendo usadas para identificar e fortalecer a competência do profissional, cortar custos e atrair clientes, atestando para um nível mais avançado de desempenho.

Lysaghta e Altschuldb [12] compreendem a certificação como um dos pilares para a competência do profissional de TI, que se relaciona com conhecimento, habilidade, padrões, competências e sua avaliação, obsolescência. A união destes itens é que dá a medida da competência do profissional. Os conceitos dos autores podem ser vistos no quadro 1.

\begin{tabular}{|l|l|}
\hline Conceito & Definição \\
\hline Conhecimento & $\begin{array}{l}\text { Identificações, diferenciações, conceitos, classificações, regras, princípios, } \\
\text { processos, operações e estratégias }\end{array}$ \\
\hline Habilidade & A proficiente aplicação do conhecimento em um processo ou tarefa \\
\hline Padrões & $\begin{array}{l}\text { O nível de conhecimento e habilidade que deve ser demonstrada para } \\
\text { apresentar um desempenho competente }\end{array}$ \\
\hline Competência & $\begin{array}{l}\text { O grau em que cada indivíduo usa o conhecimento, habilidade e } \\
\text { julgamentos associados com o campo de domínio da prática profissional }\end{array}$ \\
\hline $\begin{array}{l}\text { Avaliação da conhecimentos e } \\
\text { competência }\end{array}$ & $\begin{array}{l}\text { Avaliação da performance de efetiva aplicação de conhecion padrão de } \\
\text { habilidades fixadas no trabalho }\end{array}$ \\
\hline Obsolescência & $\begin{array}{l}\text { A discrepância entre o nível profissional de proficiência e o padional } \\
\text { estado da arte corrente no campo de trabalho }\end{array}$ \\
\hline Certificação & O reconhecimento formal de uma competência técnica de um profissiona \\
\hline
\end{tabular}

\section{Quadro 1: Conceitos centrais relacionados com a competência profissional}

A idéia exposta no quadro 1 é tangenciada por [4], que citam o expertise, a certificação e a autonomia como elementos que constituem uma profissão. Para os autores, o profissional geralmente adquire expertise em um campo do conhecimento através de uma educação formal e a aplicação do conhecimento aprendido. Já a certificação é o método para estimar o expertise de um profissional através de um instrumento de medida padronizado, e a certificação profissional é um meio para afunilar a escolha e, assim, simplificar o processo intrinsecamente problemático de selecionar um expert. A autonomia é como o elemento de discrição dado a um profissional no acompanhamento de suas tarefas, dando ao profissional um certo poder e prestígio, uma vez que este retém o controle sobre seu próprio trabalho.

Para McKillip e Cox [14], "a certificação profissional está crescendo de importância no ambiente de trabalho", sendo usada para "identificar e fortalecer a competência dos funcionários, cortar custos e atrair clientes". A certificação é obtida por indivíduos de que demonstram um domínio definido de um conjunto de conhecimentos necessários para o 
sucesso em determinada atividade, para separar os que adquiriram conhecimento apenas da prática (sabem o que já fizeram) dos que conseguem buscar outros conhecimentos [12].

A certificação profissional traz uma série de benefícios ao profissional, entre os quais pode-se citar:

a) a certificação é uma forma prática do profissional avaliar suas habilidades e experiência, é uma auto-avaliação em relação aos altos padrões da indústria [8; 29];

b) auxilia no avanço da carreira $[5,8]$, possibilitando mais chances em processos seletivos [6];

c) ser certificado é ser membro de um distinto grupo de profissionais [8], o que confere reconhecimento e satisfação ao profissional $[5,6]$;

d) o profissional certificado resolve rapidamente os problemas do gestor de TI [13];

e) o profissional certificado se sente mais confiante na execução das atividades [6];

f) a certificação é uma garantia de qualidade dos serviços prestados pelo profissional certificado $[6,9,29]$;

g) a certificação é um mecanismo motivador, pois impele o profissional a aprender novas habilidades, para obter novas certificações [9, 13];

h) o fabricante do software, bem como as empresas que utilizam este software, reconhecem o conhecimento do profissional através da sua certificação [22];

i) a certificação também provê ao profissional certificado um suporte e assistência da instituição que o certificou $[20,22]$. Este suporte auxilia no dia-a-dia e possibilita que o profissional continue aprendendo enquanto coloca em prática a sua certificação [22];

j) o profissional certificado pode obter melhores salários e benefícios $[5,6,15,20]$;

1) o profissional pode utilizar logotipia do fabricante, o que confere credibilidade [22];

Porém, tantos benefícios são acompanhados de alguns investimentos, seja de tempo, dinheiro ou perseverança. A seguir são listados alguns custos aos quais o processo de certificação está submetido:

a) o investimento financeiro para obter uma certificação é significativo [5], e nem sempre é custeado pela empresa na qual o profissional trabalha [16];

b) além do custo dos exames, há o custo do material didático e dos cursos necessários à certificação [16];

c) o profissional precisa investir tempo para obter a sua certificação [5], e em alguns casos é necessário também experiência na ferramenta [15];

d) há um certo desgaste psicológico implícito no processo de certificação, e também um certo incômodo, devido à preocupação com aulas, prazos, testes, etc [5].

\subsection{Tipos, níveis e benefícios da certificação}

De acordo com Pierson, Frolick e Chen [20], há na literatura centenas de possibilidades de certificação. No entanto, há diferentes tipos e enfoques atribuídos à expressão 'certificação de TI', tais como:

a) certificação de produtos de TI: é também chamada de certificação de TI, embora o seu enfoque seja os produtos e serviços da área. Baseia-se em testes, que comparam se o produto está em conformidade com o padrão estabelecido [30];

b) certificação de desenvolvimento de software, utilizando práticas como o CMM (Capability Maturity Model) ou as estabelecidas pela ISO e ABNT [27];

c) certificação digital, utilizada na área de segurança de informações, para assegurar que o emissor da mensagem é realmente quem diz tê-la emitido [10];

d) a certificação profissional, objeto deste artigo.

A certificação profissional é um processo estruturado, que envolve desde o fabricante até o profissional certificado. Para o fabricante, é importante ter profissionais certificados, 
pois estes elevam a importância da tecnologia em que foram certificados e mantém o seu produto na mídia, enfim, forma-se uma cadeia de valor da certificação, conforme exposto na figura 1.

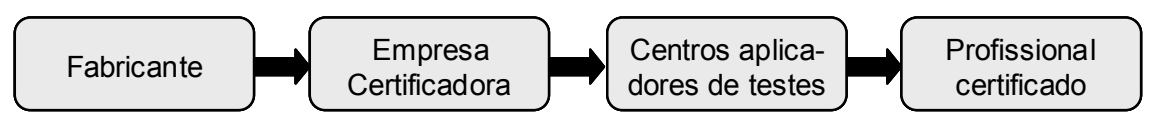

Figura 1: Cadeia de valor da Certificação

Fonte: baseado em [15] e [16]

O elo inicial da cadeia é o fabricante (Microsoft, Oracle, Cisco, etc). Como o fabricante não quer envolver-se no processo de certificação (não é sua core competence), ele nomeia empresas certificadoras (como a Vue e a Prometric). As empresas certificadoras desenvolvem e corrigem os testes, de acordo com conteúdos definidos junto ao fabricante (e sob supervisão deste), e repassam aos centros aplicadores de testes (testing centers), que têm como tarefa a aplicação dos testes de certificação junto ao elo final da cadeia, o profíssional certificado.

A fim de facilitar a compreensão da cadeia de certificação, far-se-á uso de um exemplo: a Microsoft (fabricante) necessita de profissionais certificados para uma nova ferramenta. A Microsoft entra em contato com a Vue e Prometric (empresas certificadoras), e juntas elas elaboram o conteúdo no qual o profissional será avaliado (os testes a serem aplicados pelas empresas certificadoras são sempre distintos entre si). A partir deste conteúdo, a Vue e Prometric desenvolvem os testes e avisam os centros certificadores (empresas como Processor, Fasttraining, Open, ACERT, entre outras) de que um novo teste de certificação está disponível via empresa certificadora. Os fabricantes divulgam em seus sites o novo teste, bem como a forma e o calendário de aplicação de exames, para que o profissional da área possa realizá-lo. A figura 2, abaixo, ilustra o processo de certificação.

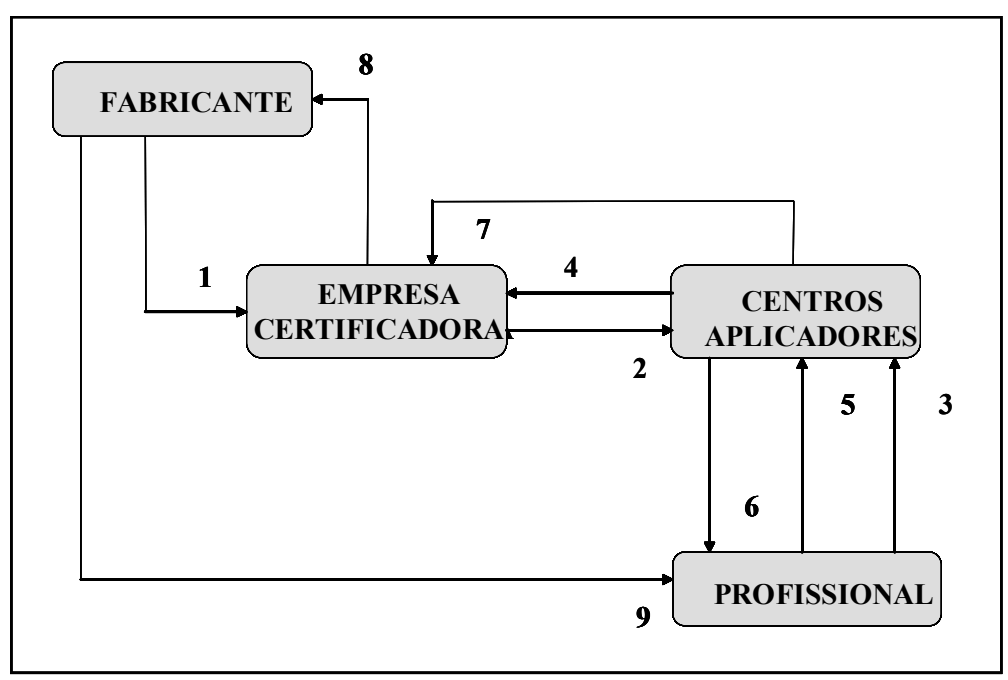

Figura 2: Processo de certificação

No primeiro momento do processo de certificação, o fabricante envia os conteúdos e/ou questões para a empresa certificadora (etapa 1), anuncia em seu site a nova certificação e avisa a empresa certificadora, que por sua vez avisa o centro aplicador (etapa 2). $\mathrm{O}$ profissional interessado então dirige-se a um dos centros aplicadores para efetuar sua inscrição junto a empresa certificadora e a marcação do respectivo exame (etapa 3), que posteriormente encaminha as informações para a empresa certificadora (etapa 4). No dia 
marcado para a realização do exame, a empresa certificadora encaminha a prova por meio eletrônico para o centro aplicador (etapa 4) onde o profissional irá realizar a prova (etapa 5). Ao final do exame, o resultado é imediatamente fornecido para o profissional (etapa 6) e para a empresa certificadora (etapa 7). Caso o candidato tenha sido aprovado, a empresa certificadora encaminha as informações necessárias para o fabricante (etapa 8), que fornecerá o certificado ao profissional (etapa 9), concluindo o processo de certificação.

O teste é realizado pelo profissional na sede do centro aplicador de testes. Em geral, os testes são feitos de forma presencial, sob rígido esquema de segurança quanto a fraudes (o profissional é filmado e permanece sob observação durante a realização da prova). Mesmo o teste sendo presencial, a prova geralmente é eletrônica, com questões pré-formatadas de múltipla escolha, fornecendo automaticamente, ao final, o escore do candidato [2].

\subsection{Carreira, mercado de trabalho e empregabilidade do profissional de TI}

McKillip e Cox [14] citam que estudos recentes indicam a certificação como critério decisivo em um processo de seleção, por ser um importante componente de trabalho de um profissional de TI. De fato, atualmente são exigidos novos requisitos para a qualificação do trabalhador [21], uma vez que a revolução provocada pela economia digital intensificou as possibilidades de automação industrial e traz à tona o debate sobre emprego, qualificações e relações industriais [28].

Desta forma, têm-se um novo cenário de trabalho. Em paralelo a esta exigência de qualificação observa-se, também como tendência global, um aumento na terceirização da produção, através da qual as empresas de grande porte deram início a um esforço de enxugamento, concentrando-se no seu core business, visando responder com rapidez às necessidades e demandas do mercado [23].

Esta realidade tem se mostrado mais relevante ainda para o profissional de TI, onde a terceirização tem crescido rapidamente, tendo-se tornado "uma realidade e vista como uma alternativa estratégica para as empresas" [25, p.1]. Desta forma, tais transformações passaram a demandar do profissional novos requisitos e atributos (pessoais, comportamentais e de conhecimento), como forma de melhorar a sua qualificação, aumentando o seu índice de empregabilidade [21]. Neste cenário, a certificação adquire mais importância, pois quando uma organização decide terceirizar parte de suas operações de TI, ela precisa ter um mecanismo de aferição dos conhecimentos do profissional terceirizado, uma vez que este estará atuando diretamente em um dos ativos mais importantes da organização: a informação. Com a certificação, "o indivíduo assegura prontidão para pôr em prática uma profissão" [17].

\section{Método de pesquisa}

Este trabalho é de natureza exploratório-descritiva. Exploratória, pois busca compreender como ocorre um determinado comportamento, elencando novas possibilidades de pesquisa [24], e descritiva porque tem como objetivo descrever características de determinada população ou fenômeno e o estabelecimento de relações entre as variáveis [1]. A estratégia de pesquisa adotada foi o estudo de caso [31], sendo que a coleta de dados ocorreu através de análise de sites e de documentos.

O caso estudado é a certificação profissional de TI, tendo como unidade de análise cada tipo de certificação (pode haver vários tipos para cada empresa). A amostra analisada compôs-se de 16 empresas: Borland, Checkpoint, Cisco, Cyclades, IBM, ISS, Java, Linux, McAFEE,Microsoft, Módulo, Norton, Novell, Oracle, Sun, Unix. O critério de seleção foi certificações de TI relacionadas a software que estivessem listadas no site das empresas certificadoras Vue (www.vue.com) e Prometric (www.prometric.com). A localização destas informações foi feita nos seguintes conjuntos de sites: fabricantes da tecnologia (Microsoft, 
Oracle, etc.); empresas certificadoras (como VUE e Prometric); centros aplicadores de testes (Brás e Figueiredo, Impacta, 4Linux, etc); publicações da área de TI (InfoExame, Computer World e IDG); busca em MCPMAG [15, 16]. Cabe ressaltar que quando havia inconsistência entre duas fontes, buscava-se uma terceira para esclarecer tal informação, sendo esta fonte uma publicação, contato com profissionais da área e mesmo a experiência dos autores na área de TI.

O protocolo utilizado na coleta de dados compôs-se das dez variáveis: empresa certificadora, nome da certificação, objeto da certificação (redes, desenvolvimento de aplicações, administração de sistemas operacionais, banco de dados, segurança, programação, administração de servidores, profissionais de help desk, programação web), público-alvo, nível (básico, intermediário, avançado), treinamento (quantidade de módulos, quantidade de horas e custo), exames (quantidade de exames e custo), salário médio do profissional certificado, informações adicionais. Os dados coletados deram origem a um banco de dados que foi analisado com o auxílio de um software específico para análise de dados, através de medidas de tendência central, agrupamentos, regressão múltipla e clusters.

\section{Resultados: características do mercado de certificação}

A partir da análise dos sites, conforme explicitado no item 3, foram selecionadas certificações em tecnologias de 16 empresas, totalizando 77 tipos de certificações, conforme tabela 1.

Tabela 1: Tecnologias certificadas

\begin{tabular}{l|l|l|l|l|l}
\hline Tecnologia certificada & Citações & $\mathbf{\%}$ & Tecnologia certificada & Citações & $\%$ \\
\hline Cisco & 14 & $18,2 \%$ & Novell & 4 & $5,2 \%$ \\
\hline Microsoft & 10 & $13,0 \%$ & Oracle & 3 & $3,9 \%$ \\
\hline Unix & 9 & $11,7 \%$ & Checkpoint & 3 & $3,9 \%$ \\
\hline Cyclades & 5 & $6,5 \%$ & Norton & 3 & $3,9 \%$ \\
\hline Java & 5 & $6,5 \%$ & ISS & 3 & $3,9 \%$ \\
\hline Borland & 5 & $6,5 \%$ & Sun & 2 & $2,6 \%$ \\
\hline McAFEE & 5 & $6,5 \%$ & IBM & 1 & $1,3 \%$ \\
\hline Linux & 4 & $5,2 \%$ & Módulo & 1 & $1,3 \%$ \\
\hline \multicolumn{7}{l}{ TOTAL $=77$ observações $(100 \%)$} & \multicolumn{3}{l}{} \\
\hline
\end{tabular}

A maior quantidade de certificações diferentes foi Cisco, Microsoft e Unix (de 9 a 14 certificações). No entanto, o número de certificações em cada tipo não é um fator decisivo, uma vez que cabe a cada empresa definir em quantos tipos de certificação dividirá o conjunto de conhecimentos necessários para que um profissional de TI possa utilizar adequadamente os seus produtos.

Outro ponto analisado foi o objeto da certificação, conforme exposto na tabela 2.

O maior número de certificações se concentrou na área de redes, seguido de desenvolvimento de aplicações, administração de sistemas operacionais e segurança. Percebese o impulso dado pela Internet para o mercado de redes, onde se encontram (além de LAN's, VAN's, VPN's e redes wireless) roteadores e protocolo TCP/IP. As certificações em segurança provavelmente também foram impulsionadas pela Internet, além dos ISP's (Internet Service Provider), ASP's (Application Service Provider) e outros xSP's, categoria genérica que denomina os serviços fornecidos através da Internet [11].

As certificações sobre administração de sistemas operacionais provavelmente foram bastante impulsionadas pelo Linux, cujo crescimento tem sido grande. Já o desenvolvimento de aplicações, que sempre foi uma das principais razões de existência da TI, se mantém com boa quantidade de citações. 
Tabela 2: Objeto das certificações

\begin{tabular}{|l|l|l|}
\hline Objeto da certificação & Citações & $\mathbf{\%}$ \\
\hline Redes (exceto segurança de redes) & 36 & $46,8 \%$ \\
\hline Segurança & 10 & $13,0 \%$ \\
\hline Administração de sistemas operacionais & 10 & $13,0 \%$ \\
\hline Desenvolvimento de aplicações & 10 & $13,0 \%$ \\
\hline Banco de dados & 2 & $2,6 \%$ \\
\hline Administração de servidores & 4 & $5,2 \%$ \\
\hline Profissionais de help desk & 2 & $2,6 \%$ \\
\hline Programação web & 2 & $2,6 \%$ \\
\hline Programação & 1 & $1,3 \%$ \\
\hline TOTAL OBS. & 77 & $100 \%$ \\
\hline
\end{tabular}

Em relação ao nível das certificações, 19 se referem a conhecimentos avançados dos certificandos, 48 de nível intermediário e 10 de nível básico. Em relação aos módulos de treinamento, estes vão de 1 a 12 , predominando 2 módulos ou 1 módulo (em 25 e 15 certificações, respectivamente), 4 módulos (14 certificações) ou 3 módulos (12 certificações). As demais 11 certificações tem 5, 6 e 7 módulos, respectivamente em 5, 3 e 2 certificações, sendo que apenas 1 certificação tem 12 módulos de treinamento. Já a exigência formal de treinamento ocorre conforme o exposto na tabela 3.

Tabela 3: Horas de treinamento

\begin{tabular}{|l|l|l|}
\hline Treinamento em horas & Citações & \% \\
\hline Até 40 & 16 & $20,8 \%$ \\
\hline de 41 a 80 & 25 & $32,5 \%$ \\
\hline de 81 a 120 & 14 & $18,2 \%$ \\
\hline de 121 a 160 & 12 & $15,6 \%$ \\
\hline de 161 a 200 & 4 & $5,2 \%$ \\
\hline de 201 a 240 & 4 & $5,2 \%$ \\
\hline Acima de 240 & 2 & $2,6 \%$ \\
\hline TOTAL OBS. & 77 & $100 \%$ \\
\hline
\end{tabular}

Os treinamentos vão de 8 a 280 horas, com uma média de 103,4 horas (desvio padrão de 64,35), sendo a maioria deles até 80 horas de duração. No entanto, observa-se uma quantidade razoável de treinamentos com mais de 120 horas (22 certificações), relacionados mais a certificações sobre redes e segurança.

O número de exames para cada certificação vai de 1 a 7 , predominando 1 e 2 exames (47 certificações, 61,1\%), seguido de 4 exames (14 certificações), 3 exames ( 8 certificações), 5 (4 certificações), 6 ( 1 certificações), 7 (2 certificações). As certificações com quantidade mais alta de exames se referem a redes, segurança, desenvolvimento de aplicações e banco de dados.

Os exames têm custos bastante diferentes, de $\mathrm{R} \$ 90,00$ a $\mathrm{R} \$ 5.400,00$ (média $=\mathrm{R} \$$ $507,48$; desvio-padrão $=\mathrm{R} \$ 706,70)$. A maior parte das certificações não tem um custo elevado (54 exames custam até R\$500,00), conforme a tabela 4. 
Tabela 4: Custo dos exames

\begin{tabular}{|l|l|l|}
\hline Custo dos exames (em R\$) & Citações & \% \\
\hline Até 300 & 29 & $37,7 \%$ \\
\hline de 301 a 500 & 26 & $33,8 \%$ \\
\hline de 501 a 750 & 15 & $19,5 \%$ \\
\hline de 751 a 1000 & 4 & $5,2 \%$ \\
\hline de 1001 a 2000 & 0 & $0,0 \%$ \\
\hline de 2001 a 3000 & 2 & $2,6 \%$ \\
\hline Acima de 3000 & 1 & $1,3 \%$ \\
\hline TOTAL OBS. & 77 & $100 \%$ \\
\hline
\end{tabular}

Os exames acima de $\mathrm{R} \$ 1.000,00$ são voltados para redes e administração de servidores. Se analisarmos a tabela 4 juntamente com a 5, pode-se concluir que o investimento tem retorno, pois o salário médio de um profissional certificado é atrativo: de R\$ $2.500,00$ a $\mathrm{R} \$ 10.000,00$ (média $=\mathrm{R} \$ 4.442,03$; desvio-padrão $=\mathrm{R} \$ 1.464,12$ ), conforme tabela 5 .

Tabela 5: Salário médio do certificado

\begin{tabular}{|l|l|l|}
\hline Salário médio do certificado (em R\$) & Citações & \% \\
\hline Não resposta & 8 & $10,4 \%$ \\
\hline Até 3000 & 18 & $23,4 \%$ \\
\hline de 3001 a 4000 & 16 & $20,8 \%$ \\
\hline de 4001 a 5000 & 22 & $28,6 \%$ \\
\hline de 5001 a 7000 & 10 & $13,0 \%$ \\
\hline Acima de 7000 & 3 & $3,9 \%$ \\
\hline TOTAL OBS. & 77 & $100 \%$ \\
\hline
\end{tabular}

O maior número de certificações (22) se concentra na faixa salarial de até R\$ 5.000,00, seguida de Até R \$ 3.000,00 (18 certificações) e R \$ 3.001,00 a R \$ 4.000,00 (16 certificações). Há uma quantidade expressiva de certificações com remuneração entre R\$ 5.001,00 e R \$ 7.000,00 (10 certificações, de redes, segurança, desenvolvimento de aplicações e programação web). Três certificações têm renda acima de $\mathrm{R} \$ 7.000$ ( 2 de redes e 1 de administração de sistemas operacionais). As 8 não-respostas se referem a remuneração por hora ao invés de mensal (de $\mathrm{R} \$ 150,00$ a $\mathrm{R} \$ 250,00$ para consultores sênior), não podendo ser analisados em conjunto com os dados da tabela 5.

A tabela 6 traça um panorama das certificações em relação a treinamento em horas, número de exames, custo do treinamento/exames e salário médio do profissional certificado. Os valores nas colunas se referem à média de todas as certificações de cada empresa.

Se observarmos os valores em negrito em cada linha da tabela 6 (que são os valores superiores à média, exposta na última linha), podemos identificar uma certa relação de custobenefício. Por exemplo, a certificação da Cisco exige do candidato a certificado muitas horas de treinamento, além de vários exames e alto investimento, mas o salário é atrativo. No entanto, a certificação da IBM tem um custo bem mais elevado de treinamento (em relação à Cisco) e oferece um salário menor.

Ainda neste mesmo raciocínio, utilizou-se a técnica de clusters para tentar identificar se havia grupos de certificações que aliassem alto custo e alto benefício, e inversamente menor custo e menor benefício. Para tanto, foram utilizadas as variáveis (numéricas) Treinamento em horas, Número de exames, Custo do treinamento, Custo dos exames e 
Salário médio do certificado, as 4 primeiras representando o investimento e a última representando o retorno da certificação. A recomendação estatística [7] é que a técnica de clusters seja utilizada a partir de 100 casos, mas procedeu-se aqui com 77 casos, devendo-se, portanto, considerar que as conclusões obtidas com esta técnica são preliminares.

Através da classificação automática feita pelo software SPHINX®, obteve-se 5 clusters, no entanto, um deles tinha baixa representatividade. Conforme indica a bibliografia sobre o assunto, rodou-se novamente a técnica, agora delimitando em 4 clusters, que ficaram com boa representatividade $(13,14,20$ e 22 citações) e confiabilidade (os percentuais de explicação entre as variáveis de cada cluster foram de 62,4\% até 96\%).

Tabela 6: Panorama das certificações

\begin{tabular}{|l|r|r|r|r|r|}
\hline Variáveis & $\begin{array}{c}\text { Treinamento } \\
\text { em horas } \\
\text { (Média) }\end{array}$ & $\begin{array}{c}\text { Número de } \\
\text { exames } \\
\text { (Média) }\end{array}$ & $\begin{array}{c}\text { Custo do } \\
\text { treinamento - } \\
\text { em R\$ (Média) }\end{array}$ & $\begin{array}{c}\text { Custo dos } \\
\text { exames - em } \\
\text { R\$ (Média) }\end{array}$ & $\begin{array}{c}\text { Salário médio } \\
\text { do certificado - } \\
\text { em R (Média) }\end{array}$ \\
\hline Cisco (14) & $\mathbf{1 3 5 , 4 3}$ & $\mathbf{3 , 3 6}$ & $\mathbf{1 3 . 5 9 2 , 8 6}$ & $\mathbf{7 3 1 , 7 9}$ & $\mathbf{5 . 2 1 4 , 2 9}$ \\
\hline Microsoft (10) & $\mathbf{1 1 4 , 0 0}$ & $\mathbf{3 , 1 0}$ & $4.493,20$ & 223,50 & $\mathbf{4 . 2 0 0 , 0 0}$ \\
\hline Unix (9) & $\mathbf{1 0 9 , 3 3}$ & 1,33 & $\mathbf{7 . 1 8 6 , 8 9}$ & $\mathbf{6 4 0 , 0 0}$ & $4.000,00$ \\
\hline McAFEE (5) & 68,80 & 1,80 & $1.890,00$ & 360,00 & valor por hora \\
\hline Borland (5) & $\mathbf{1 2 8 , 0 0}$ & $\mathbf{3 , 2 0}$ & $2.720,00$ & 375,00 & $3.500,00$ \\
\hline Java (5) & 81,60 & 1,40 & $5.107,00$ & 435,00 & $\mathbf{5 . 2 0 0 , 0 0}$ \\
\hline Cyclades (5) & 6,00 & 1,00 & 506,40 & 100,00 & $2.900,00$ \\
\hline Linux (4) & 85,50 & 1,00 & $2.750,00$ & 331,50 & $\mathbf{4 . 6 2 5 , 0 0}$ \\
\hline Novell (4) & $\mathbf{1 4 0 , 0 0}$ & $\mathbf{3 , 7 5}$ & $3.975,00$ & 250,00 & $4.375,00$ \\
\hline Oracle (3) & 36,00 & $\mathbf{3 , 6 7}$ & $6.021,33$ & 375,00 & $\mathbf{5 . 0 0 0 , 0 0}$ \\
\hline Norton (3) & $\mathbf{1 1 7 , 3 3}$ & $\mathbf{5 , 6 7}$ & $\mathbf{8 . 0 6 6 , 6 7}$ & $\mathbf{2 . 1 0 0 , 0 0}$ & $\mathbf{4 . 8 3 3 , 3 3}$ \\
\hline ISS (3) & 80,00 & 2,00 & $\mathbf{2 0 . 0 0 0 , 0 0}$ & $\mathbf{7 5 0 , 0 0}$ & valor por hora \\
\hline Checkpoint (3) & 18,67 & 2,00 & $3.383,33$ & 350,00 & $4.000,00$ \\
\hline Sun (2) & $\mathbf{1 4 0 , 0 0}$ & 2,50 & $\mathbf{8 . 7 0 0 , 0 0}$ & 300,00 & $4.000,00$ \\
\hline IBM (1) & $\mathbf{1 1 2 , 0 0}$ & $\mathbf{4 , 0 0}$ & $\mathbf{2 1 . 0 0 0 , 0 0}$ & 285,00 & $\mathbf{5 . 0 0 0 , 0 0}$ \\
\hline Módulo (1) & $\mathbf{1 2 0 , 0 0}$ & 1,00 & $\mathbf{8 . 5 5 0 , 0 0}$ & $\mathbf{5 5 0 , 0 0}$ & $\mathbf{7 . 0 0 0 , 0 0}$ \\
\hline $\begin{array}{l}\text { Média do } \\
\text { conjunto (77) }\end{array}$ & 103,40 & 2,55 & $\mathbf{6 . 9 7 7 , 8 6}$ & 507,48 & $4.442,03$ \\
\hline
\end{tabular}

A tabela 7, a seguir, mostra o detalhamento do cluster, sendo que o valor da célula representa a média de valores e o valor entre parênteses representa a ordem, do maior para o menor (por exemplo, 1 representa o cluster com maior número de exames e 4 representa o cluster com menor número de exames, e assim sucessivamente).

Tabela 7: Clusters identificados

\begin{tabular}{|l|r|r|r|r|r|}
\hline Yariáveis & $\begin{array}{c}\text { Treinamento } \\
\text { em horas }\end{array}$ & $\begin{array}{c}\text { Número } \\
\text { de exames }\end{array}$ & $\begin{array}{c}\text { Custo do } \\
\text { treinamento } \\
\text { (em R\$) }\end{array}$ & $\begin{array}{c}\text { Custo dos } \\
\text { exames (em } \\
\text { R\$) }\end{array}$ & $\begin{array}{c}\text { Salário médio } \\
\text { do certificado } \\
\text { (em R\$) }\end{array}$ \\
\hline Cluster 3 & $181,54(1)$ & $4,38(1)$ & $17.984,62(1)$ & $1.232,31(1)$ & $5.653,85(1)$ \\
\hline Cluster 2 & $127,45(2)$ & $2,82(2)$ & $6.874,68(2)$ & $412,95(2)$ & $5.181,82(2)$ \\
\hline Cluster 4 & $77,40(3)$ & $2,25(3)$ & $3.327,85(3)$ & $334,00(3)$ & $3.775,00(3)$ \\
\hline Cluster 1 & $47,57(4)$ & $1,21(4)$ & $1.160,36(4)$ & $231,50(4)$ & $3.107,14(4)$ \\
\hline
\end{tabular}

Os clusters se mostraram bastante homogêneos, pois em todos eles a combinação de números (de 1-1-1-1-1 a 4-4-4-4-4) foi igual. Sendo assim, parece-nos que o cluster 3 é o de 
mais investimento e maior retorno, seguido do cluster 2,4 e 1 , sendo este último o de menor investimento e menor retorno.

\begin{tabular}{|c|c|c|c|}
\hline \multirow{5}{*}{$\begin{array}{l}\text { Maior } \\
\text { investimento, } \\
\text { maior retorno }\end{array}$} & Cluster & Tecnologia certificada & Objeto da certificação \\
\hline & 3 & $\begin{array}{l}\text { Unix, Cisco, Microsoft, IBM e } \\
\text { Norton }\end{array}$ & $\begin{array}{l}\text { Administração de servidores, } \\
\text { Redes (exceto segurança de redes) } \\
\text { Segurança } \\
\text { Banco de dados }\end{array}$ \\
\hline & 2 & $\begin{array}{l}\text { Linux, Sun, Cisco, Novell, } \\
\text { Oracle, Java, Microsoft, } \\
\text { Borland, Módulo }\end{array}$ & $\begin{array}{l}\text { Administração de SO } \\
\text { Administração de servidores } \\
\text { Redes (exceto segurança de redes) } \\
\text { Segurança } \\
\text { Desenvolvimento de aplicações } \\
\text { Programação web }\end{array}$ \\
\hline & 4 & $\begin{array}{l}\text { Microsoft, Unix, Cisco, Novell, } \\
\text { Oracle, Java, Microsoft, } \\
\text { Borland, Checkpoint, Norton }\end{array}$ & $\begin{array}{l}\text { Redes (exceto segurança de redes) } \\
\text { Segurança } \\
\text { Administração de SO } \\
\text { Profissionais de help desk } \\
\text { Programação } \\
\text { Desenvolvimento de aplicações }\end{array}$ \\
\hline & 1 & $\begin{array}{l}\text { Microsoft, Linux, Cyclades, } \\
\text { Java, Borland, Norton }\end{array}$ & $\begin{array}{l}\text { Redes } \\
\text { Administração de SO } \\
\text { Desenvolvimento de aplicações } \\
\text { Programação web }\end{array}$ \\
\hline $\begin{array}{l}\text { Menor } \\
\text { investimento, } \\
\text { menor retorno }\end{array}$ & & & \\
\hline
\end{tabular}

Figura 3: Custo-benefício e identificação de cada cluster

O fato de algumas empresas (como Cisco e Microsoft) aparecerem em diferentes clusters não caracteriza uma incoerência metodológica, uma vez que isso se deve ao fato de elas terem um grande número de certificações (14 e 10, respectivamente, representando $31,2 \%$ das 77 certificações analisadas), e, naturalmente, um leque de certificações variado, inclusive em relação ao custo/benefício.

Finalizando, procurou-se verificar se o salário médio do certificado era função de outros valores, tais como o investimento em qualificação, procedendo-se com o teste de regressão múltipla, conforme a tabela 8 .

Tabela 8: Variáveis que possivelmente explicam o salário de um profissional certificado

\begin{tabular}{|l|c|}
\hline Variáveis explicativas & Salário médio do certificado \\
\hline Treinamento em módulos & 0,55 \\
\hline Treinamento em horas & 0,57 \\
\hline Número de exames & 0,52 \\
\hline Custo do treinamento & 0,54 \\
\hline Custo dos exames & 0,39 \\
\hline
\end{tabular}

Os valores de correlação entre as variáveis explicativas com o salário médio não foram elevados (quanto mais próximas de 1, maior a correlação entre cada variável explicativa com a variável a explicar), explicando juntas $41,7 \%$ da variação de salário médio. Embora quando retiradas as variáveis 'custo de treinamento' e 'custo dos exames' o percentual de explicação 
reduza, revelando certa importância deste ao conjunto, as variáveis acima pouco explicam o salário médio do certificado. Sendo assim, outros fatores não quantitativos, como experiência, confiança, cargos ocupados, etc., podem influenciar o salário do profissional certificado.

\section{Considerações finais}

Este artigo pôde confirmar a necessidade e importância dos programas de certificação profissional, bem como uma positiva relação custo-benefício. Ao analisarmos a tabela 7 e a figura 3, vemos que a certificação é um investimento de retorno garantido, e que pode ser planejada e galgada passo a passo pelo profissional de TI.

Para os profissionais de TI, este artigo pode ser útil como uma visão da certificação, com alguns detalhes técnicos, de custo e de relação custo-benefício. Para os gestores de TI, vale lembrar que são novos tempos, que exigem um novo tipo de profissional, e que a certificação pode ser um critério importante na seleção e contratação de pessoal. Naturalmente, ela não elimina que outros aspectos (pessoais e comportamentais) sejam abordados. Também é necessário refletir sobre a estrutura dos programas de capacitação promovidos ou incentivados pela empresa. Para as universidades, este artigo pode contribuir para uma reflexão sobre a formação dos egressos em áreas de TI. Conforme citam Mulkeym e O'Neil JR. [17], assuntos como certificação, transferência de habilidades, complexidade do trabalho e da mão-de-obra devem ser a vanguarda de preocupações educacionais, como forma de não ficar em desvantagem em relação ao mercado.

Este artigo não pretende esgotar o debate sobre o tema, que é importante para que organizações públicas e privadas adquiram melhores níveis de produtividade e, com isso, contribuam com a maturidade da área de TI em nosso país. Pelo contrário, o tema carece ainda de muitas investigações, sugerindo-se como pesquisas futuras: a) submeter os resultados aqui encontrados à apreciação de pessoas que contratam profissionais de TI, verificando as necessidades e benefícios de um profissional certificado; b) confrontar os resultados aqui obtidos, que são uma visão da empresa certificadora e da fabricante de tecnologia (uma vez que a pesquisa foi feita em sites e publicações destas) com a visão dos profissionais certificados; c) uma pesquisa confirmatória que possa identificar variáveis explicativas para o salário do profissional certificado, uma vez que, como mostra a tabela 8 , só o processo de certificação não foi suficiente para isto; d) uma pesquisa exploratória que verifique outros benefícios (mais qualitativos) de ser um profissional certificado. Conforme citado no item 2.1, estes benefícios podem ser: reconhecimento do mercado, credibilidade, rápido avanço na carreira, status, melhores oportunidades de emprego, satisfação profissional, entre outros.

Além disso, conforme sugere Voas [29], é importante certificar os três pilares (produto, processo e pessoal) da qualidade de software em uma empresa com certificação da maturidade do processo de software, com software desenvolvido e testado por profissionais certificados, e este software, como produto, submetido a certos padrões de qualidade, deveria obter um melhor resultado, podendo ser comprovado através de um estudo da maior confiança e qualidade do software produzido caso apenas um dos pilares fosse certificado.

\section{Referências}

[1] BICKMAN, Leonard, ROG, Debra J., HEDRICK, Terry. E. Applied research design: a practical approach. In: Bickman, Leonard. e Rog, Debra. J. (org). Handbook of applied social research methods. Thousand Oaks: Sage Publications, 1997.

[2] BLAIR, D.V., O'NEIL JR., H.F., PRICE, D.J. Effects of expertise on state self-efficacy and state worry during a computer-based certification test. Computers in Human Behavior, 1999, n. 15, p. 511-530.

[3] CASE, Thomas A., BOTELHO, Joaquim M. Gerenciamento da carreira do executivo 
brasileiro: uma ciência exata. São Paulo: CATHO, 2001.

[4] CEGIELSKI, Casey G., REBMAN, Carl M., REITHEL, Brian J. The value of certification: an empirical assessment of the perceptions of end-users of local area networks. In: Information Systems Journal, n. 13, p. 97-107, 2003.

[5] CHURCHILL JR, Gilbert A., PETER, J. Paul. Marketing: criando valor para os clientes. São Paulo: Saraiva, 2000.

[6] FURLAN, Alessandra Fabris. Oracle Certified Professional: avaliação dos diferenciais resultantes da certificação. Pós-Graduação em Gestão Empresarial. PPGA/EA/UFRGS. Porto Alegre: UFRGS, 2000.

[7] HAIR, Joseph F. et al. Multivariate data analysis. New Jersey: Prentice-Hall, 1998. 5. ed. [8] ICCP (Institute for Certified Computer profissionals). Benefits of Certification. Disponível em http://www/iccp.org/benefits. Acessado em 21-julho-2003.

[9] ISSAC, G.; RAJENDRAN, C.; ANANTHARAMAN, R. N. Significance of quality certification: the case of the software industry in India. The Quality Management Journal, 2004, v. 11, n. 1, pp. 8-32.

[10] KAKABADSE-KORAC, Nada; KAKABADSE, Andrew. IS/IT Governance: Need for an integrated model. Corporate Governance. MCB University Press, 1, 4, p. 9-11. 2001.

[11] LUCIANO, Edimara Mezzomo, FREITAS, Henrique. Comércio eletrônico de produtos virtuais: a Internet modificando a operação de comprar e vender produtos. São Paulo: Anais do VI SIMPOI, outubro de 2003, anais em CD-ROM.

[12] LYSAGHTA, Rosemary M., ALTSCHULDB, James W. Beyond initial certification: the assessment and maintenance of competency in professions. Evaluation and Program Planning, 2000, n. 23, p. 95-104.

[13] MCGRATH, S. T. The future of IT Certification. Training \& Development, v.52, n.6, junho 1998, p. 18-25.

[14] MCKILLIP, Jack, COX, Caryl. Strengthening the criterion-related validity of professional certifications. Evaluation and Programming Planning, 1998, n. 21, p.191-197.

[15] MCPMAG - Microsoft Certified Professional Magazine Online - Salary Survey 2002. Disponível em http://mcpmag.com/salarysurveys. Acessado em 21-julho-2003a.

[16] MCPMAG - Microsoft Certified Professional Magazine Online - Salary Survey 2002. Disponível em http://mcpmag.com/salarysurveys/article.asp?editorialsid=59. Acessado em 21-jul-2003b.

[17] MULKEYM, J. R., O'NEIL JR, H.F. The effects of test item format on self-efficacy and worry during a high-stakes computer-based certification examination. Computers in Human Behavior, 1999, n. 15, p. 495-509.

[18] MURRY, J. P. Sucessfully Hiring and Retaining IT Personnel. Information Systems Management, Spring 1999.

[19] PRESSMAN, R. Engenharia de software. São Paulo :Makron Books, 1995.

[20] PIERSON, Leslie, FROLICK, Mark N., CHEN, Leida. Emerging issues in IT certification. The Journal of Computer Information Systems. Fall 2001, v.42, n.1, p.17-20.

[21] RESENDE, Patrícia T. D., PAULA, Germano M. de. Inovações modernizantes e novos requisitos da formação profissional: um estudo de caso na indústria automobilística brasileira. In: Anais do XXIV ENANPAD. Florianópolis: ANPAD, 2000.

[22] RODA, Roy. IT skills standards. Communications of the ACM. New York: abril 1999, v.42,n.4, p.21-26.

[23] SÁ CARVAlHO, Luis Carlos de, ESTEVES, Mônica, PARET, Benito D. Percepções de executivos de empresas de pequeno porte sobre o conhecimento, a informação e a aprendizagem: um desafio educacional. In: Anais do XXIV ENANPAD. Florianópolis: ANPAD, 2000. 
[24] SAMPIERI, Roberto et al. Metodología de la investigación. México: McGraw-Hill, 1991.

[25] SANTOS, Carlos A. P. N., OLIVEIRA, Francisco C. Terceirização no processo de desenvolvimento de sistemas de informações. In.: Anais do XXIV ENANPAD. ANPAD: 2000. [26] SAVIANI, José R. O analista de negócios e da informação. São Paulo: Atlas, 1998. $4^{\text {a }}$ ed.

[27] VAN DER PIJL, G. J., SWINKELS, G. J. P., VERRIJDT, J. G. ISO 9000 versus CMM: standardization and certification of IS development. Information \& Management, 1997, n.32, p. 267-274.

[28] VIEIRA, Adriane, GARCIA, Fernando Coutinho. O efeito das novas tecnologias sobre a qualificação dos trabalhadores: salvador ou diabólico? Porto Alegre/RS: Anais do $X X X V I I$ CLADEA, Outubro de 2002, anais em CD-ROM.

[29] VOAS, Jeffrey. The Software Quality Certification Triangle. The Journal of Defense Software Engineering, novembro/ 1998, pp. 12-14.

[30] WIECHERS, W.K. IT Certification in Europe. Computer Standards \& Interfaces, 1988, n.7, p. 169-173.

[31] YIN, Robert K. Case study research: design and methods. California: Sage Publications, 1994. 